
\title{
25 Research Sourere \\ First Report of Enteroaggregative E. Coli Harbouring NDM-4 and NDM-7 From Asymptomatic Children in India
}

\section{Jaspreet Mahindroo}

Post Graduate Institute of Medical Education and Research

\section{Vinay Modgil}

Post Graduate Institute of Medical Education and Research

\section{Pankaj Chaudhary}

Post Graduate Institute of Medical Education and Research

\section{Bhavneet Bharti}

Post Graduate Institute of Medical Education and Research

\section{Balvinder Mohan}

Post Graduate Institute of Medical Education and Research

Neelam Taneja ( $\nabla$ drneelampgi@yahoo.com )

PGIMER: Post Graduate Institute of Medical Education and Research https://orcid.org/0000-00031198-6138

\section{Research Article}

Keywords: NDM, EAEC, WGS, asymptomatic carriage, India

Posted Date: December 9th, 2021

DOl: https://doi.org/10.21203/rs.3.rs-1022546/v1

License: (c) (i) This work is licensed under a Creative Commons Attribution 4.0 International License. Read Full License 


\section{Abstract}

For the first time in the world, we report Enteroaggregative E.coli harbouring $b / a_{\mathrm{NDM}-4}$ and $b / a_{\mathrm{NDM}-7}$ that were isolated from healthy children. NDM-4 and NDM-7 are associated with increased carbapenemase activity. Clonal spread, inter-strain and interspecies horizontal transfer of such resistant determinants in a healthy population will be a cause of concern.

\section{Main Text}

New Delhi metallo-beta-lactamase (NDM) is a carbapenemase having a broad range of hydrolysing activity against all beta lactam antibiotics (except aztreonam) including carbapenems which are last resort antibiotics to treat a multitude of health-care associated infections. Since its first discovery, 20 variants of NDM have been discovered so far (1). These variants differ from NDM-1 by one to two residues at different positions of the active site of the NDM protein. NDM-4 has a single amino acid substitution (Met154Leu) while NDM-7 has double mutations [(Asp130 Asn) and (Met154Leu)], all of these mutations have been confirmed to increase the carbapenemase activity (2). Till now, only sporadic isolations of carbapenem resistant Enterobacteriaceae (CRE) carrying NDM-4 and NDM-7 variants have been reported from patients admitted to ICUs, and hospital sewage mainly from Asian countries (3-5). Commensal carriage of NDM-1 which is the most prevalent NDM type has been reported from healthy adults and children from many countries across the world (6) (). Enteroaggregative Escherichia coli (EAEC) is an evolving enteric pathogen that is significantly associated with acute and persistent diarrhoea, malnutrition in children, HIV infection, and traveller's diarrhoea (7). Asymptomatic EAEC carriage are common early in life specially in developing countries which can lead to intestinal inflammation and clinical growth shortfalls (8).

Here for the first time, we report EAEC harbouring NDM-4 and NDM-7 variants isolated from healthy children. We collected stool samples from 550 healthy children $<5$ years of age from 25 anganwadi centers of Chandigarh which offer primary healthcare to children as a part of the government public healthcare system (9). The EAEC were identified by using pCVD432 gene coding for CVD432 on pAA plasmid of EAEC (9). Whole genomes of EAEC strains $(n=33)$ were sequenced on Illumina Hiseq platform to generate $150 \times 2$ bp paired end reads and the sequences were analysed for in silico MLST, serotyping, identification of antimicrobial resistance (AMR) genes, plasmid replicons by SRST2 v0.2.0 (10). The genomes were assembled with SPADES v3.14.0 (11), annotated with RAST v2.0 and viewed on SEED genome browser v2.0 (12). The raw reads from this study are available under the BioProject ID: PRJNA760671; accession numbers: SAMN21220438 and SAMN21220439.

In two of the EAEC isolates (S56 and S76) originating respectively from a four year and a one year old female children, variants of $b / a_{N D M-1}$ gene i.e. $b_{\mathrm{NDM}_{-4}}$ and $\mathrm{bla}_{\mathrm{NDM}-7}$ were detected. Both these females had no history of antibiotic exposure or underlying conditions at the time of sample collection and were located at two different anganwadis situated in the Chandigarh city. Phenotypically both strains were multi-drug resistant (MDR) showing resistance to ampicillin, ciprofloxacin, co-trimoxazole, levofloxacin, 
cefoxitin, cefepime, ceftriaxone, ertapenem and imipenem. In addition to NDM-4, sample S56 also carried genes encoding AmpC cephalosporinases ( $a m p H$, ampC1, ampC2), various ESBLs (bla $\mathrm{CTX}-\mathrm{M}-15$, bla ${ }_{\mathrm{TEM}-206}, b_{\mathrm{OXA-1}}$ ), aminoglycosides (aac3-lla, aac6lb-cr, aadA12, sat-2A, strA, strB), chloramphenicol (catB4), trimethoprim (dfrA1, dfrA12), mrdA (encodes PBP2), rmtB (16srRNA methylase), sulphonamides (sulll, sull), and tetracycline (tetA) resistances. This strain belonged to ST6303 from clonal complex 31 with 015:H34 antigenic serotype. Additionally IncFII (pRSB107) plasmid was also identified in this genome. Plasmids of IncFIl group are widely known to be involved in the worldwide dissemination of AMR genes as in the case $b / a_{\mathrm{CTXM}-15}$ gene (13). The virulence profile of this isolate composed of signature EAEC genes such as aggACDR, air, aap, aar, aatA. The second isolate (S76) carrying NDM-7, also harboured genes encoding AmpC cephalosporinases ( $a m p H$, ampC1, ampC2), ESBLs (bla $\mathrm{CMY}-42$, $b / a_{\mathrm{CTX}-\mathrm{M}-15}$ ), and $m r d A$ (encodes PBP2) resistances. This isolate belonged to ST448 (clonal complex CC448) with 0188:H19 antigenic serotype and possessed a self-transmissible plasmid IncX3. ST448 as well as IncX3 plasmid associated with NDM variants have also been reported from clinical cases in India $(5,14,15)$. The major virulence gene present in this isolate was aar gene encoding AggR-activated regulator. In both strains, immediately upstream of $b / a_{\mathrm{NDM}}$ genes contained bleomycin resistance gene (ble) along with $d s b D$ encoding oxidoreductase and trp1 encoding putative phosphoribosylanthranilate isomerase (for synthesis of amino acid tryptophan) were observed as reported earlier from India (Figure) (5).

We believe this to be the first report in world literature where EAEC with $b / a_{\mathrm{NDM}-4}$ and $b / a_{\mathrm{NDM}-7}$ were isolated from healthy children. NDM-4 and NDM-7are both associated with increased carbapenemase activity and clonal spread of such strains, inter-strain and interspecies horizontal transfer of such resistant determinants in healthy population will be a cause of concern. The gut is a very highly populated ecosystem and at a later stage such strains may be a source of extra intestinal infections, may spread to other hosts or transfer genetic resistance element to other members of micro-biota including pathogens.

\section{Abbreviations}

NDM: New Delhi metallo-beta-lactamase

CRE: Carbapenem resistant Enterobacteriaceae

EAEC: Enteroaggregative Escherichia coli

AMR: Antimicrobial resistance

MDR: Multi-drug resistant

\section{Declarations}


The study was approved by the Postgraduate Institute of Medical Education and Research (PGIMER) Ethics Committee (INT/IEC/2017/173). Written informed consent was obtained from the parent/guardian to participate in the study. All methods were performed in accordance to the relevant guidelines and regulations of the Institute Ethics Committee.

\section{Consent to publish}

A written consent from the parent/guardian of the children were taken to publish the results. All the patient identifiers were removed and sample IDs have been coded to safeguard the patient identity.

\section{Data availability}

The raw reads generated in this study have been deposited to NCBI under BioProject ID: PRJNA760671; accession numbers: SAMN21220438 and SAMN21220439.

\section{Acknowledgement}

We acknowledge kind support of local health authorities for facilitating the sample access in their areas

\section{Funding information}

The work was supported by grant no. 5/8-1(37)/D/2012-13/ECD-II from Indian Council of Medical Research (ICMR), New Delhi

\section{Author contributions}

JM: Methodology, Investigation, Formal Analysis, Manuscript original draft, Writing - review \& editing

VM: Methodology and Investigation

PC: Resources

BB: Resources

BM: Project administration, Supervision

NT: Conceptualization, Funding acquisition, Investigation, Project administration, Supervision, Validation, Manuscript original draft, Writing - review \& editing

\section{Conflicts of interests}

None to declare

\section{First author biography}


Jaspreet Mahindroo (PhD) is a Research Associate in Department of Medical Microbiology at PGIMER, Chandigarh, India. Her research is focused on infectious disease epidemiology, antimicrobial resistance, and microbial genomics.

\section{Corresponding author}

Dr. Neelam Taneja, Professor, Department of Medical Microbiology, Research Block A, PGIMER, Chandigarh, India.+91-9780232453, drneelampgi@yahoo.com

\section{References}

1. Liu Z, Li J, Wang X, Liu D, Ke Y, Wang Y, et al. Novel Variant of New Delhi Metallo-beta-lactamase, NDM-20, in Escherichia coli. Front Microbiol. 2018;9:248.

2. Khan AU, Maryam L, Zarrilli R. Structure, Genetics and Worldwide Spread of New Delhi Metallo-betalactamase (NDM): a threat to public health. BMC Microbiol. 2017;17(1):101.

3. Ahmad N, Ali SM, Khan AU. Detection of New Delhi Metallo-beta-Lactamase Variants NDM-4, NDM-5, and NDM-7 in Enterobacter aerogenes Isolated from a Neonatal Intensive Care Unit of a North India Hospital: A First Report. Microb Drug Resist. 2018;24(2):161-5.

4. Beg AZ, Khan AU. Genome analyses of blaNDM-4 carrying ST 315 Escherichia coli isolate from sewage water of one of the Indian hospitals. Gut Pathog. 2018;10:17.

5. Devanga Ragupathi NK, Muthuirulandi Sethuvel DP, Gajendiran R, Daniel JL, Walia K, Veeraraghavan B. First Indian report of IncX3 plasmid carrying blaNDM-7 in Escherichia coli from bloodstream infection: potential for rapid dissemination. New Microbes New Infect. 2017;17:65-8.

6. Shen Z, Hu Y, Sun Q, Hu F, Zhou H, Shu L, et al. Emerging Carriage of NDM-5 and MCR-1 in Escherichia coli From Healthy People in Multiple Regions in China: A Cross Sectional Observational Study. EClinicalMedicine. 2018;6:11-20.

7. Estrada-Garcia T, Perez-Martinez I, Bernal-Reynaga R, Zaidi MB. Enteroaggregative Escherichia coli: A Pathogen Bridging the North and South. Curr Trop Med Rep. 2014;1:88-96.

8. Rogawski ET, Guerrant RL, Havt A, Lima IFN, H. P, Medeiros QS, et al. Epidemiology of enteroaggregative Escherichia coli infections and associated outcomes in the MAL-ED birth cohort. PLoS Negl Trop Dis. 2017;11(7).

9. Modgil V, Chaudhary P, Bharti B, Mahindroo J, Yousuf M, Koundal M, et al. Prevalence, Virulence Gene Profiling, and Characterization of Enteroaggregative Escherichia coli from Children with Acute Diarrhea, Asymptomatic Nourished, and Malnourished Children Younger Than 5 Years of Age in India. J Pediatr. 2021;234:106-14 e5.

10. Inouye M, Dashnow H, Raven L-A, Schultz MB, Pope BJ, Tomita T, et al. SRST2: Rapid genomic surveillance for public health and hospital microbiology labs. Genome Medicine. 2014;6:90.

11. Bankevich A, Nurk S, Antipov D, Gurevich AA, Dvorkin M, Kulikov AS, et al. SPAdes: a new genome assembly algorithm and its applications to single-cell sequencing. J Comput Biol. 2012;19(5):455- 
77.

12. Overbeek R, Olson R, Pusch GD, Olsen GJ, Davis JJ, Disz T, et al. The SEED and the Rapid Annotation of microbial genomes using Subsystems Technology (RAST). Nucleic Acids Res. 2014;42.

13. Boyd DA, Tyler S, Christianson S, McGeer A, Muller MP, Willey BM, et al. Complete nucleotide sequence of a 92-kilobase plasmid harboring the CTX-M-15 extended-spectrum beta-lactamase involved in an outbreak in long-term-care facilities in Toronto, Canada. Antimicrob Agents Chemother. 2004;48(10):3758-64.

14. Choudhury NA, Paul D, Chakravarty A, Bhattacharjee A, Dhar Chanda D. IncX3 plasmid mediated occurrence of blaNDM-4 within Escherichia coli ST448 from India. J Infect Public Health. 2018;11(1):111-4.

15. Paul D, Bhattacharjee A, Ingti B, Choudhury NA, Maurya AP, Dhar D, et al. Occurrence of blaNDM-7 within IncX3-type plasmid of Escherichia coli from India. J Infect Chemother. 2017;23(4):206-10.

\section{Figures}

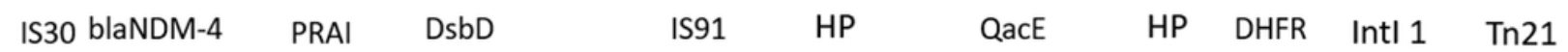

a)

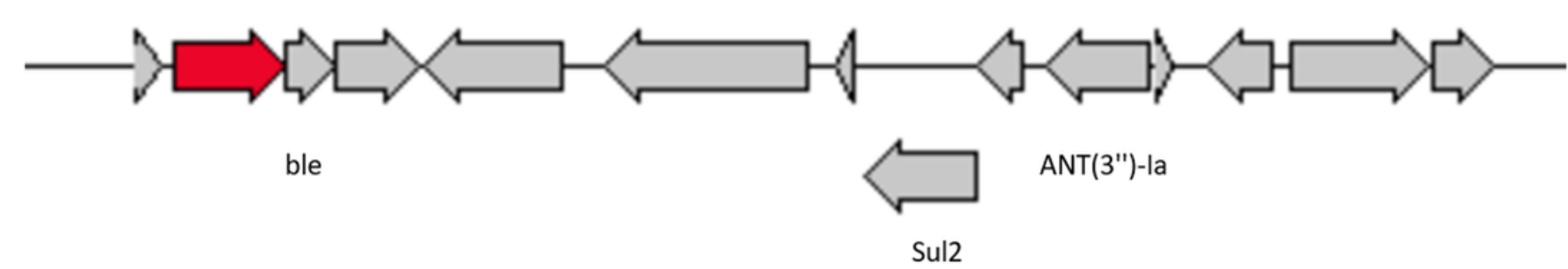

$\begin{array}{llll}\text { blaNDM-7 ble } & \text { PRAl }\end{array}$

b)

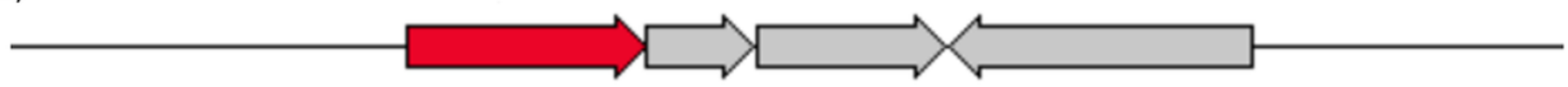

\section{Figure 1}

Schematic representation of genetic environment of blaNDM region. a. S76 and b S56; blaNDM-4 and blaNDM-7 are shown in red; ble: gene encoding bleomycin resistance protein, IS: insertion element, DsbD: oxidoreductase, trp1: gene encoding putative phosphoribosylanthranilate isomerase 